\title{
Gradient approximation and extremum seeking via needle variations *
}

\author{
Simon Michalowsky and Christian Ebenbauer **
}

October 1, 2018

\begin{abstract}
We consider a gradient approximation scheme that is based on applying needle shaped inputs. By using ideas known from the classic proof of the Pontryagin Maximum Principle we derive an approximation that reveals that the considered system moves along a weighted averaged gradient. Moreover, based on the same ideas, we give similar results for arbitrary periodic inputs. We also present a new gradient-based optimization algorithm that is motivated by our calculations and that can be interpreted as a combination of the heavy ball method and Nesterov's method.
\end{abstract}

\section{Introduction}

Extremum seeking is a well-known technique that has successfully been used in several applications (see e.g. [5], [17], [18]) in order to operate a system at an a priori unknown setpoint that is optimal with respect to some objective function. In a typical extremum seeking problem this objective function is unknown such that gradient-based methods do not apply. The majority of the extremum seeking schemes relies on approximating the gradient by the excitation of the system with a so called dither signal. Although a much larger class of periodic signals is appropriate in principle, in most cases sinusoidal dither signals are used. The effect of the choice of the dither has been studied in [15] where the authors conclude that dither signals other than sinusoidal ones could be beneficial.

In the present paper we introduce periodic needle-shaped dither signals for gradient approximation. In particular we consider the case where the period length is large compared to the pulse length. This is in contrast to standard averaging results ([2]) or Lie bracket averaging results ([7]) where the period length is also assumed to be small. Our main objective is not to present a new extremum seeking scheme here but to get insight into the extremum seeking process using needle-shaped dither signals.

\footnotetext{
* This article is an extended version of [12].

** S. Michalowsky and C. Ebenbauer are with the Institute for Systems Theory and Automatic Control, University of Stuttgart, 70550 Stuttgart, Germany. simon.michalowsky@ist.uni-stuttgart.de, ce@ist.uni-stuttgart.de. This work was supported by the German Research Foundation (DFG, EB 42S/4-1).
}

Nevertheless, this class of dithers could also be of interest in certain applications, e.g. when it is only possible to apply inputs in a short period of time.

Our contributions are as follows: For the case of needle-shaped dither signals we show that the extremum seeking system approximates a weighted averaged gradient descent. Secondly, we use that a large class of dither signals is well approximated by a summation of multiple needle-shaped signals and we show how extremum seeking systems with such dither signals can be analyzed with the presented theory as well. We establish new results for finite period length that are in the limit in accordance with existing results. We further propose a new gradient-based optimization algorithm that is indeed motivated by the results on gradient approximation via needle variations but can also be regarded separately. The algorithm can be seen as a combination of a continuous-time version of Nesterov's method and the heavy ball method and is of interest on its own.

Our analysis relies on well-known results established in the context of the Pontryagin Maximum Principle where the effects of needle-shaped variations of the optimal inputs are studied. Since the Maximum Principle is well-established in a very broad setup we hope that our ideas can be used for much more general problems. However, our results should be seen as a first step and in the present paper we only consider basic cases.

The structure of the present paper is as following: In Section 2 we give a brief introduction to needle variations and the variational equations. In Section 3 we present our main results. We first consider the case of two needles with opposite sign, then we show how this can be generalized to a superposition of many needles and third we present the new continuous-time algorithm. In Section 4 we illustrate our approximation formulas for the particular case of a quadratic objective function and give simulation results for the proposed new algorithm. We conclude our work in Section 5. 


\section{Preliminaries}

\subsection{Notation}

We denote by $\mathbb{N}$ the set of natural numbers, by $\mathbb{Z}$ the set of integer numbers and by $\mathbb{R}$ the set of real numbers. We denote by $\mathscr{C}^{n}$ the set of $n$ times continuously differentiable functions. The gradient of a function $f: \mathbb{R}^{p} \rightarrow \mathbb{R}, f \in \mathscr{C}^{1}$ is denoted by $\nabla f(x)=\left[\begin{array}{llll}\frac{\partial f}{\partial x_{1}}(x) & \frac{\partial f}{\partial x_{2}}(x) & \ldots & \frac{\partial f}{\partial x_{p}}(x)\end{array}\right]^{\top}$. Moreover, we make use of the Landau notation: For $f, g: \mathbb{R}^{n} \rightarrow \mathbb{R}$ we write $f(x)=$ $\mathscr{O}(g(x))$ meaning that there exists some $M>0$ and some $\delta>0$ such that $|f(x)| \leq M|g(x)|$ for all $|x| \leq \delta$.

\subsection{Needle variations and the variational equation}

In the following we briefly repeat well-known results on the effect of so called needle variations in the control inputs to trajectories of dynamic systems. Our overview follows closely the lines of [11, Chapter 4] but is adapted to the special needs for the argumentation of our main results. Consider

$$
\dot{x}(t)=g_{1}(x(t)) u_{1}(t)+g_{2}(x(t)) u_{2}(t)
$$

where $g_{1}, g_{2}: \mathbb{R} \rightarrow \mathbb{R}$ and $u_{1}, u_{2}: \mathbb{R} \rightarrow \mathbb{R}$. Further, suppose that $g_{1}, g_{2} \in \mathscr{C}^{1}$ and $u_{1}, u_{2}$ are piecewise continuous such that at least local existence and uniqueness of a solution to (1) is ensured. Let $x^{*}(t)$ denote the solution of (1) for $u_{1}(t)=u_{1}^{*}(t)$ and $u_{2}(t)=u_{2}^{*}(t) \equiv 0$. We are interested in how the solution $x^{*}(t)$ will change when we perturb the input $u_{2}^{*}$ by a so called needle variation, also known as Pontryagin-McShane variation, where the perturbed input is defined as

$$
u_{2}(t)= \begin{cases}u_{2}^{*}(t) & \text { if } t \notin[\bar{t}-\varepsilon, \bar{t}] \\ \alpha & \text { if } t \in[\bar{t}-\varepsilon, \bar{t}]\end{cases}
$$

with some constant $\alpha \in \mathbb{R}$ and $\varepsilon>0$. Thus, the input is perturbed on an interval of length $\varepsilon$ by some constant value $\alpha$, see Figure 1. In the following we will investigate the effect of such a perturbation for small $\varepsilon$. Let $x(t)$ denote the solution of (1) when applying the perturbed input and suppose that $u_{1}^{*}(t)$ is continuous at $t=\bar{t}$. By some Taylor expansions one can show that (see e.g. [11])

$$
\begin{aligned}
& x(\bar{t})=x^{*}(\bar{t})+\varepsilon[ g_{1}\left(x^{*}(\bar{t})\right) u_{1}^{*}(\bar{t})+g_{2}\left(x^{*}(\bar{t})\right) \alpha \\
&\left.-g_{1}\left(x^{*}(\bar{t})\right) u_{1}^{*}(\bar{t})\right]+\mathscr{O}\left(\varepsilon^{2}\right) \\
&=x^{*}(\bar{t})+\varepsilon g_{2}\left(x^{*}(\bar{t})\right) \alpha+\mathscr{O}\left(\varepsilon^{2}\right) .
\end{aligned}
$$

We will now investigate how the perturbed solution $x(t)$ is propagated after time $\bar{t}$ in comparison to the unperturbed solution $x^{*}(t)$. This can be studied using perturbation theory ([9]) where one is interested in how the solution of a differential equation evolves when starting from a perturbed initial condition compared to the solution when starting from a nominal initial condition. Here, $x^{*}(\bar{t})$ plays the role of the nominal initial condition whereas the perturbed one is given by (3). The basic idea is to do a Taylor expansion of the perturbed solution in $\varepsilon$ about $\varepsilon=0$ which leads to

$$
x(t)=x^{*}(t)+\varepsilon v_{1}(t)+\mathscr{O}\left(\varepsilon^{2}\right)
$$

where $v_{1}(t)$ is some unknown function that can be determined as following. Since $x(t)$ must fulfill the unperturbed differential equation one can put $x(t)$ as given by (4) into (1) with $u_{1}=u_{1}^{*}$, $u_{2}=u_{2}^{*}$ and compare the terms linear in $\varepsilon$ which then gives ([11],[9])

$$
\dot{v}_{1}(t)=\frac{\partial g_{1}}{\partial x}\left(x^{*}(t)\right) u_{1}^{*}(t) v_{1}(t)
$$

with $v_{1}(\bar{t})=g_{2}\left(x^{*}(\bar{t})\right) \alpha$. This is known as the variational equation ([11]) and it is the same as the linearization of (1) with $u_{1}(t)=u_{1}^{*}(t), u_{2}(t)=u_{2}^{*}(t)$ about the trajectory $x^{*}(t)$.

\section{Main results}

We consider the following nonlinear input-affine system

$$
\dot{x}(t)=F(x(t)) u_{1}(t)+u_{2}(t)
$$

with $x(0)=x_{0} \in \mathbb{R}$ and where $F: \mathbb{R} \rightarrow \mathbb{R}, F \in \mathscr{C}^{1}$. In extremum seeking problems the usual approach is to choose the inputs $u_{1}, u_{2}$ such that the trajectories of (6) approximately move along those of the gradient flow $\dot{\bar{x}}(t)=-\nabla F(\bar{x}(t)), \bar{x}(0)=x_{0}$, such that $x$ converges to a solution of the optimization problem $\min F(x)$ (see e.g. [2] or [7]). Here, we want to investigate the averaged behavior of (6) for a new class of inputs: We first consider needle-shaped inputs and then give a generalization thereof. We consider the scalar case here such that, strictly speaking, we are only treating derivative approximation. However it should be noted that one can expect similar results for the multidimensional case.

\subsection{Dither signals composed of two needles}

Define the following $T$-periodic input sequence

$$
\begin{aligned}
& u_{1}(t)= \begin{cases}1 & \text { for } t \in\left[0, \frac{T}{2}\right) \\
-1 & \text { for } t \in\left[\frac{T}{2}, T\right)\end{cases} \\
& u_{2}(t)= \begin{cases}\alpha & \text { for } t \in[0, \varepsilon) \\
0 & \text { for } t \in\left[\varepsilon, \frac{T}{2}\right) \\
-\alpha & \text { for } t \in\left[\frac{T}{2}, \frac{T}{2}+\varepsilon\right) \\
0 & \text { for } t \in\left[\frac{T}{2}+\varepsilon, T\right)\end{cases}
\end{aligned}
$$

where $T>0,0<\varepsilon<\frac{T}{2}$ and $-\infty<\alpha<\infty$. The input sequence is illustrated in Figure 2. The following theorem reveals how this input sequence affects (6) for $\varepsilon$ being small. 


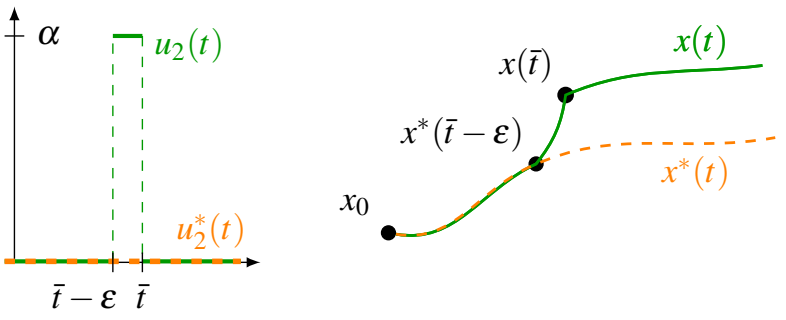

Figure 1. Illustration of the needle variation as defined by (2) (left) as well as the optimal and the varied trajectory (right).
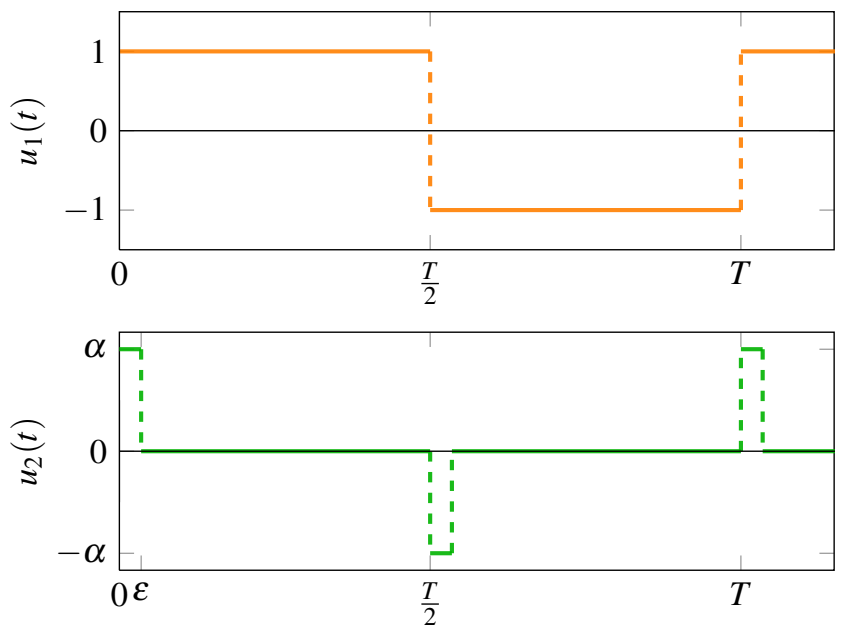

Figure 2. Illustration of the input sequence as defined by (7) and (8). The upper plot shows $u_{1}(t)$, the lower one shows the needle variation $u_{2}(t)$.

Theorem 1. Consider (6) together with the input sequence as defined by (7) and (8). Let $x^{*}(t)$ denote the solution of (6) when $u_{1}(t)$ as defined by (7) and $u_{2}(t) \equiv 0$ and suppose that $x^{*}(t)$ exists on $[0, T]$. Let $\Phi\left(t, t_{0}\right)$ denote the state-transition matrix at time $t$ corresponding to the variational equation

$$
\dot{v}_{1}(t)=\frac{\partial F}{\partial x}\left(x^{*}(t)\right) v_{1}(t)
$$

with initial time $t_{0}$. Then

$$
\begin{aligned}
& x(T)= \\
& x_{0}+\varepsilon \alpha \Phi(0, \varepsilon) \int_{\varepsilon}^{\frac{T}{2}-\varepsilon} \frac{\partial F}{\partial x}\left(x^{*}(\tau)\right) \Phi\left(\tau, \frac{T}{2}-\varepsilon\right) d \tau+\mathscr{O}\left(\varepsilon^{2}\right) .
\end{aligned}
$$

Proof. We use the results presented in Section 2.2 where $u_{2}$ plays the role of the needle variation. With (3) we have after the first needle, i.e. at $t=\varepsilon, x(\varepsilon)=x^{*}(\varepsilon)+\varepsilon \alpha+\mathscr{O}\left(\varepsilon^{2}\right)$. Using (4) we have at the point where the second needle is applied, i.e. at $t=\frac{T}{2}$,

$$
x\left(\frac{T}{2}\right)=x^{*}\left(\frac{T}{2}\right)+\varepsilon v_{1}(t)+\mathscr{O}\left(\varepsilon^{2}\right)
$$

where $v_{1}(t)$ is the solution of (9) with initial condition $v_{1}(\varepsilon)=\alpha$. Since $\Phi\left(t, t_{0}\right)$ denotes the state-transition matrix corresponding to (9) we have that $v_{1}(t)=\Phi(t, \varepsilon) v_{1}(\varepsilon)=\Phi(t, \varepsilon) \alpha$ for $t \in\left[\varepsilon, \frac{T}{2}\right]$ such that

$$
x\left(\frac{T}{2}\right)=x^{*}\left(\frac{T}{2}\right)+\varepsilon \Phi\left(\frac{T}{2}, \varepsilon\right) \alpha+\mathscr{O}\left(\varepsilon^{2}\right) .
$$

We will now repeat the same procedure for the second needle. Let $\bar{x}(t)$ denote the solution when only applying the first but not the second needle, i.e. we have $\bar{x}(t)=x^{*}(t)+\varepsilon \bar{\Phi}(t, \varepsilon) \alpha+\mathscr{O}\left(\varepsilon^{2}\right)$ where $\bar{\Phi}\left(t, t_{0}\right)$ denotes the state-transition matrix corresponding to

$$
\dot{v}(t)=\frac{\partial F}{\partial x}\left(x^{*}(t)\right) u_{1}(t) v(t)
$$

with initial time $t_{0}$. In comparison to the previous calculations $\bar{x}(t)$ now plays the role of $x^{*}(t)$ in (3) such that

$$
x\left(\frac{T}{2}+\varepsilon\right)=\bar{x}\left(\frac{T}{2}+\varepsilon\right)-\varepsilon \alpha+\mathscr{O}\left(\varepsilon^{2}\right) .
$$

Hence, propagating the second needle, we obtain by (4)

$$
\begin{aligned}
x(t) & =\bar{x}(t)+\varepsilon v_{2}(t)+\mathscr{O}\left(\varepsilon^{2}\right) \\
& =x^{*}(t)+\varepsilon\left(\bar{\Phi}(t, \varepsilon) \alpha+v_{2}(t)\right)+\mathscr{O}\left(\varepsilon^{2}\right)
\end{aligned}
$$

for $\frac{T}{2}+\varepsilon \leq t \leq T$. Let $v(t)=\bar{\Phi}(t, \varepsilon) \alpha+v_{2}(t)$. Notice that $v(t)$ fulfills (13) such that

$$
\begin{aligned}
x(T) & =x^{*}(T)+\varepsilon \bar{\Phi}\left(T, \frac{T}{2}+\varepsilon\right) v\left(\frac{T}{2}+\varepsilon\right)+\mathscr{O}\left(\varepsilon^{2}\right) \\
& =x^{*}(T)+\varepsilon \bar{\Phi}\left(T, \frac{T}{2}+\varepsilon\right)\left(\bar{\Phi}\left(\frac{T}{2}+\varepsilon, \varepsilon\right) \alpha-\alpha\right)+\mathscr{O}\left(\varepsilon^{2}\right) .
\end{aligned}
$$

We will now investigate how $\bar{\Phi}\left(t, t_{0}\right)$ and $\Phi\left(t, t_{0}\right)$ are related. Let $\Phi_{2}\left(t, t_{0}\right)$ denote the state-transition matrix corresponding to (13) when $u_{1}(t)=-1$ and notice that $\Phi\left(t, t_{0}\right)$ is the state-transition matrix for the case of $u_{1}(t)=1$. Then we have

$$
\bar{\Phi}\left(t, t_{0}\right)= \begin{cases}\Phi\left(t, t_{0}\right) & \text { if } t \in\left[0, \frac{T}{2}\right), t_{0} \in\left[0, \frac{T}{2}\right] \\ \Phi\left(t, \frac{T}{2}\right) \Phi_{2}\left(\frac{T}{2}, t_{0}\right) & \text { if } t \in\left[0, \frac{T}{2}\right], t_{0} \in\left[\frac{T}{2}, T\right] \\ \Phi_{2}\left(t, \frac{T}{2}\right) \Phi\left(\frac{T}{2}, t_{0}\right) & \text { if } t \in\left[\frac{T}{2}, T\right], t_{0} \in\left[0, \frac{T}{2}\right) \\ \Phi_{2}\left(t, t_{0}\right) & \text { if } t \in\left[\frac{T}{2}, T\right], t_{0} \in\left[\frac{T}{2}, T\right]\end{cases}
$$

Now, since (13) is a scalar linear time varying differential equation, we have $\Phi\left(t, t_{0}\right)=\exp \left(\int_{t_{0}}^{t} \frac{\partial F}{\partial x}\left(x^{*}(\tau)\right) d \tau\right.$. Similarly

$$
\begin{aligned}
& \Phi_{2}\left(t, t_{0}\right) \\
& =\exp \left(-\int_{t_{0}}^{t} \frac{\partial F}{\partial x}\left(x^{*}(\tau)\right) d \tau=\exp \left(-\int_{t_{0}}^{t} \frac{\partial F}{\partial x}\left(x^{*}(T-\tau)\right) d \tau\right.\right. \\
& =\exp \left(\int_{T-t_{0}}^{T-t} \frac{\partial F}{\partial x}\left(x^{*}(s)\right) d s=\Phi\left(T-t, T-t_{0}\right)\right.
\end{aligned}
$$

where we used that $x^{*}(t)=x^{*}(T-t)$ since $x^{*}(t)$ is just going back and forth along $F(x)$. Using (17) and (18) in (16) we obtain 
with $x^{*}(T)=x_{0}$

$$
\begin{aligned}
x(t)= & x_{0}+\varepsilon \alpha\left(\Phi_{2}\left(T, \frac{T}{2}\right) \Phi\left(\frac{T}{2}, \varepsilon\right)-\Phi_{2}\left(T, \frac{T}{2}+\varepsilon\right)\right)+\mathscr{O}\left(\varepsilon^{2}\right) \\
= & x_{0}+\varepsilon \alpha\left(\Phi(0, \varepsilon)-\Phi\left(0, \frac{T}{2}-\varepsilon\right)\right)+\mathscr{O}\left(\varepsilon^{2}\right) \\
= & x_{0}+\varepsilon \alpha \Phi(0, \varepsilon)\left(1-\Phi\left(\varepsilon, \frac{T}{2}-\varepsilon\right)\right)+\mathscr{O}\left(\varepsilon^{2}\right) \\
= & x_{0}+\varepsilon \alpha \Phi(0, \varepsilon) \int_{\varepsilon}^{\frac{T}{2}-\varepsilon} \frac{\partial}{\partial \tau} \Phi\left(\tau, \frac{T}{2}-\varepsilon\right) d \tau+\mathscr{O}\left(\varepsilon^{2}\right) \\
= & x_{0}+\varepsilon \alpha \Phi(0, \varepsilon) \int_{\varepsilon}^{\frac{T}{2}-\varepsilon} \frac{\partial F}{\partial x}\left(x^{*}(\tau)\right) \Phi\left(\tau, \frac{T}{2}-\varepsilon\right) d \tau \\
& +\mathscr{O}\left(\varepsilon^{2}\right)
\end{aligned}
$$

where in the last step we used the differentiation property of the state-transition matrix, see e.g [8].

Remark 1. Equation (10) gives an approximation at $t=T$. However, due to the periodicity, it can as well be extended to arbitrary integer multiples of $T$ which leads to

$$
\begin{aligned}
& x((k+1) T)= \\
& x(k T)+\varepsilon \alpha \Phi\left(k T, t_{k 1}\right) \int_{t_{k 1}}^{t_{k 2}} \frac{\partial F}{\partial x}\left(x^{*}(\tau)\right) \Phi\left(\tau, t_{k 2}\right) d \tau+\mathscr{O}\left(\varepsilon^{2}\right)
\end{aligned}
$$

where $k \in \mathbb{N}, t_{k 1}:=k T+\varepsilon, t_{k 2}:=k T+\frac{T}{2}-\varepsilon$.

Eq. (10) gives insight into the gradient approximation process and shows that system (6) with inputs (7) and (8) approximately moves along a weighted average of the gradient of $F$. Thus, depending on the sign of $\alpha$, this system does a modified gradient descent or ascent. This is also comparable to what is done in stochastic approximation ([13]) where one uses the sum of several gradients.

Notice that the approximation (10) is valid for small $\varepsilon$ but $T$ does not have to be small which is in contrast to existing results. In the following we consider the case where $T$ is of the same order of magnitude as $\varepsilon$. In particular, we consider $T=8 \varepsilon$.

Lemma 1. Consider (6) together with the input sequence as defined by (7) and (8). Let $x^{*}(t)$ denote the solution of (6) when $u_{1}(t)$ as defined by (7) and $u_{2}(t) \equiv 0$. Suppose $T=8 \varepsilon$. Then

$$
x(T)=x_{0}+\varepsilon^{2} \alpha\left(\frac{\partial F}{\partial x}\left(x^{*}\left(\frac{T}{2}\right)\right)+\frac{\partial F}{\partial x}\left(x_{0}\right)\right)+\mathscr{O}\left(\varepsilon^{3}\right) .
$$

Proof. In case of $T=8 \varepsilon$ we have from (10)

$$
x(T)=x_{0}+\varepsilon \alpha \Phi(0, \varepsilon) \int_{\varepsilon}^{3 \varepsilon} \frac{\partial F}{\partial x}\left(x^{*}(\tau)\right) \Phi(\tau, 3 \varepsilon) d \tau+\mathscr{O}\left(\varepsilon^{2}\right) .
$$

Expanding the integrand into a Taylor series about $\tau=4 \varepsilon$ we obtain

$$
\begin{aligned}
& \int_{\varepsilon}^{3 \varepsilon} \frac{\partial F}{\partial x}\left(x^{*}(\tau)\right) \Phi(\tau, 3 \varepsilon) d \tau \\
= & \int_{\varepsilon}^{3 \varepsilon} \frac{\partial F}{\partial x}\left(x^{*}(4 \varepsilon)\right) \Phi(4 \varepsilon, 3 \varepsilon)+\mathscr{O}(\tau-4 \varepsilon) d \tau \\
= & 2 \varepsilon \frac{\partial F}{\partial x}\left(x^{*}(4 \varepsilon)\right) \Phi(4 \varepsilon, 3 \varepsilon)+\mathscr{O}\left(\varepsilon^{2}\right) .
\end{aligned}
$$

Moreover, by a Taylor expansion of $\Phi\left(4 \varepsilon, t_{0}\right)$ about $t_{0}=4 \varepsilon$, it is

$$
\Phi(4 \varepsilon, 3 \varepsilon)=\Phi(4 \varepsilon, 4 \varepsilon)-\frac{d}{d t_{0}} \Phi\left(4 \varepsilon, t_{0}\right)_{\mid t_{0}=4 \varepsilon} \varepsilon+\mathscr{O}\left(\varepsilon^{2}\right) .
$$

Equivalently, we have

$$
\Phi(0, \varepsilon)=\Phi(0,0)+\frac{d}{d t_{0}} \Phi\left(0, t_{0}\right)_{\mid t_{0}=0} \varepsilon+\mathscr{O}\left(\varepsilon^{2}\right) .
$$

Hence we obtain using (23) - (25) in (22)

$$
x(T)=x_{0}+2 \varepsilon^{2} \alpha \frac{\partial F}{\partial x}\left(x^{*}(4 \varepsilon)\right)+\mathscr{O}\left(\varepsilon^{3}\right) .
$$

By (11) it is $x^{*}(4 \varepsilon)=x\left(\frac{T}{2}\right)+\mathscr{O}(\varepsilon)$ but also $x^{*}(4 \varepsilon)=x_{0}+\mathscr{O}(\varepsilon)$. Thus

$$
\begin{aligned}
x(T) & =x_{0}+2 \varepsilon^{2} \alpha \frac{\partial F}{\partial x}\left(x\left(\frac{T}{2}\right)\right)+\mathscr{O}\left(\varepsilon^{3}\right) \\
& =x_{0}+2 \varepsilon^{2} \alpha \frac{\partial F}{\partial x}\left(x_{0}\right)+\mathscr{O}\left(\varepsilon^{3}\right)
\end{aligned}
$$

such that - by adding both expressions - we finally arrive at (21).

Remark 2. Lemma 1 is consistent with the well-known fact that an input-affine system $\dot{x}=g_{1}(x) u_{1}(t)+g_{2}(x) u_{2}(t)$ with $u_{1}$, $u_{2}$ as defined by (7) and (8) where $T=8 \varepsilon$ approximately moves into the direction of the Lie bracket $\left[g_{1}, g_{2}\right](x)$, see e.g. [4] or [7] in terms of extremum seeking. Here, it is $g_{1}(x)=F(x)$ and $g_{2}(x)=1$ such that we obtain for the Lie bracket

$$
\left[g_{1}, g_{2}\right](x)=\frac{\partial g_{2}}{\partial x}(x) g_{1}(x)-\frac{\partial g_{1}}{\partial x}(x) g_{2}(x)=-\frac{\partial F}{\partial x}(x) .
$$

Remark 3. In case of $T=4 \varepsilon$ the integral in (10) vanishes such that there are no first order terms. Thus, the ratio between $\varepsilon$ and $T$ is important but nevertheless $T$ does not necessarily have to be $8 \varepsilon$.

In comparison to common approximation formulas our result (21) not only includes the gradient of $F$ evaluated at the start point but also at the point where $u_{1}$ changes its sign, i.e. at the two points where $x^{*}$ reverses its direction. Usually, this term disappears by including it in the higher order terms.

\subsection{Dither signals composed of infinitely many needles}

Up to now we have considered the very special input sequence given by (7) and (8). In the following we consider the same nonlinear input-affine system (6) with more general inputs $u_{1}$ 


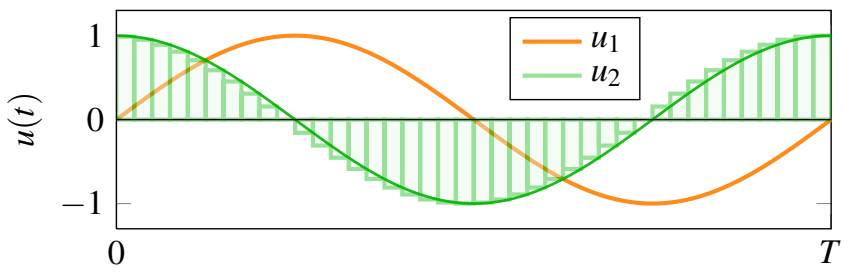

Figure 3. Illustration of the approximation of the input function $u_{1}$ by many needles as used in Theorem 2.

and $u_{2}$. More precisely, we impose the following assumptions on the input functions $u_{1}, u_{2}: \mathbb{R} \rightarrow \mathbb{R}$ :

A1 The functions $u_{1}, u_{2}$ are piecewise continuous and bounded.

A2 The functions $u_{1}, u_{2}$ are $T$-periodic and have zero mean.

For example, this includes the well-known case of trigonometric input functions, i.e. $u_{1}(t)=\sqrt{\omega} \cos (\omega t)$ and $u_{2}(t)=\sqrt{\omega} \sin (\omega t)$ that was considered e.g. in [10] or more recently in [7] in the context of extremum seeking. Loosely spoken, the idea is to approximate the input $u_{2}$ by infinitely many needles to go along the same lines as in the previous case, see Figure 3.

Theorem 2. Suppose that Assumptions A1 and A2 hold. Consider (6) together with $u_{2}(t)$ defined by

$$
u_{2}(t)=u_{2}\left(t_{i+1}\right) \quad \text { for } t \in\left[t_{i}, t_{i+1}\right), t_{i+1} \leq T
$$

where $t_{i}=\varepsilon i, \varepsilon=\frac{T}{N}, N \in \mathbb{N}, i=0,1, \ldots, N-1$. Let $x^{*}(t)$ denote the solution of (6) for $u_{2}(t) \equiv 0$ and $u_{1}(t)$ as defined by Assumptions $\mathrm{A} 1$ and $\mathrm{A} 2$ and suppose that $x^{*}(t)$ exists on $[0, T]$. Let further $\Phi\left(t, t_{0}\right)$ denote the state-transition matrix corresponding to

$$
\dot{v}_{1}(t)=\frac{\partial F}{\partial x}\left(x^{*}(t)\right) u_{1}(t) v_{1}(t)
$$

with initial time $t_{0}$. Then, if $N$ is finite,

$$
\begin{aligned}
& x(T)=x^{*}(T)+\mathscr{O}\left(\varepsilon^{2}\right) \\
& +\varepsilon \sum_{i=0}^{N-1}\left(\int_{t_{i+1}}^{T} \frac{\partial F}{\partial x}\left(x^{*}(\tau)\right) u_{1}(\tau) \Phi\left(\tau, t_{i+1}\right) d \tau+1\right) u_{2}\left(t_{i+1}\right) .
\end{aligned}
$$

Moreover,

$$
\lim _{N \rightarrow \infty} x(T)=x^{*}(T)+\int_{0}^{T} \int_{t}^{T} \frac{\partial F}{\partial x}\left(x^{*}(\tau)\right) u_{1}(\tau) \Phi(\tau, t) u_{2}(t) d \tau d t .
$$

Proof. We use again the results presented in Section 2.2. With (3) we have at the end of the first needle $x(\varepsilon)=x^{*}(\varepsilon)+\varepsilon u_{2}(\varepsilon)+$
$\mathscr{O}\left(\varepsilon^{2}\right)$. Let $\bar{x}(t)$ denote the solution in case we would not apply the second needle at $t=\varepsilon$. Then

$$
\begin{aligned}
\bar{x}(2 \varepsilon) & =x^{*}(2 \varepsilon)+\varepsilon v_{1}(2 \varepsilon)+\mathscr{O}\left(\varepsilon^{2}\right) \\
& =x^{*}(2 \varepsilon)+\varepsilon \Phi(2 \varepsilon, \varepsilon) u_{2}(\varepsilon)+\mathscr{O}\left(\varepsilon^{2}\right)
\end{aligned}
$$

and hence when the second needle is applied we obtain using again (3)

$$
\begin{aligned}
x(2 \varepsilon) & =\bar{x}(2 \varepsilon)+\varepsilon u_{2}(2 \varepsilon)+\mathscr{O}\left(\varepsilon^{2}\right) \\
& =x^{*}(2 \varepsilon)+\varepsilon \Phi(2 \varepsilon, \varepsilon) u_{2}(\varepsilon)+\varepsilon u_{2}(2 \varepsilon)+\mathscr{O}\left(\varepsilon^{2}\right) .
\end{aligned}
$$

Here, the first term involving the state-transition matrix is the propagation of the first needle whereas the other term linear in $\varepsilon$ is the second needle. Going on along these lines we obtain

$$
\begin{aligned}
x(3 \varepsilon)= & x^{*}(3 \varepsilon)+\Phi(3 \varepsilon, 2 \varepsilon)\left(\Phi(2 \varepsilon, \varepsilon) \varepsilon u_{2}(\varepsilon)+\varepsilon u_{2}(2 \varepsilon)\right) \\
& +\varepsilon u_{1}(3 \varepsilon)+\mathscr{O}\left(\varepsilon^{2}\right) \\
= & x^{*}(3 \varepsilon)+\varepsilon \Phi(3 \varepsilon, \varepsilon) u_{2}(\varepsilon)+\varepsilon \Phi(3 \varepsilon, 2 \varepsilon) u_{2}(2 \varepsilon) \\
& +\varepsilon u_{2}(3 \varepsilon)+\mathscr{O}\left(\varepsilon^{2}\right) .
\end{aligned}
$$

Generalizing this we have

$$
x(k \varepsilon)=x^{*}(k \varepsilon)+\varepsilon \sum_{i=0}^{k-1} \Phi\left(k \varepsilon, t_{i+1}\right) u_{2}\left(t_{i+1}\right)+\mathscr{O}\left(\varepsilon^{2}\right)
$$

where $k \in \mathbb{N}$ and $t_{i}=\varepsilon i$. Thus, we have after one period, i.e. at $T=N \varepsilon$,

$$
x(T)=x^{*}(T)+\varepsilon \sum_{i=0}^{N-1} \Phi\left(T, t_{i+1}\right) u_{2}\left(t_{i+1}\right)+\mathscr{O}\left(\varepsilon^{2}\right) .
$$

We will now take a closer look at the sum. It is

$$
\begin{aligned}
& \varepsilon \sum_{i=0}^{N-1} \Phi\left(T, t_{i+1}\right) u_{2}\left(t_{i+1}\right) \\
= & \varepsilon \sum_{i=0}^{N-1}\left(\int_{t_{i+1}}^{T} \frac{\partial \Phi}{\partial \tau}\left(\tau, t_{i+1}\right) d \tau+1\right) u_{2}\left(t_{i+1}\right) \\
= & \varepsilon \sum_{i=0}^{N-1}\left(\int_{t_{i+1}}^{T} \frac{\partial F}{\partial x}\left(x^{*}(\tau)\right) u_{1}(\tau) \Phi\left(\tau, t_{i+1}\right) d \tau+1\right) u_{2}\left(t_{i+1}\right)
\end{aligned}
$$

where we used in the last step that the state-transition matrix $\Phi$ fulfills the variational equation (30). This, together with (37), proves (31). Next we look at the case when $N$ tends to infinity. We consider first the part without he integral, i.e.

$$
\lim _{N \rightarrow \infty} \sum_{i=0}^{N-1} \varepsilon u_{2}\left(t_{i+1}\right)=\lim _{N \rightarrow \infty} \sum_{i=0}^{N-1}\left(t_{i+1}-t_{i}\right) u_{2}\left(t_{i+1}\right) .
$$

This is the limit of a Riemann sum (see e.g [16, Chapter 3]) with 
partition $\left\{t_{i}\right\}$ such that

$$
\lim _{N \rightarrow \infty} \sum_{i=0}^{N-1} \varepsilon u_{2}\left(t_{i+1}\right)=\int_{0}^{T} u_{2}(t) d t .
$$

Since $u_{2}$ has zero mean by Assumption A2, this integral vanishes. For the second part of the sum we calculate similarly

$$
\begin{aligned}
& \lim _{N \rightarrow \infty} \sum_{i=0}^{N-1} \int_{t_{i+1}}^{T} \frac{\partial F}{\partial x}\left(x^{*}(\tau)\right) u_{1}(\tau) \Phi\left(\tau, t_{i+1}\right) \varepsilon u_{2}\left(t_{i+1}\right) d \tau \\
= & \lim _{N \rightarrow \infty} \sum_{i=0}^{N-1} \int_{t_{i+1}}^{T} \frac{\partial F}{\partial x}\left(x^{*}(\tau)\right) u_{1}(\tau) \Phi\left(\tau, t_{i+1}\right)\left(t_{i+1}-t_{i}\right) u_{2}\left(t_{i+1}\right) d \tau \\
= & \int_{0}^{T} \int_{t}^{T} \frac{\partial F}{\partial x}\left(x^{*}(\tau)\right) u_{1}(\tau) \Phi(\tau, t) u_{2}(t) d \tau d t
\end{aligned}
$$

where we once more used that we have a Riemann sum here that converges to the Riemannian integral.

Remark 4. Theorem 2 also applies with a slight modification if Assumption A2 is not fulfilled. However, periodicity is required to extend the approximation as explained in Remark 1. Further, the mean of $u_{2}(t)$ appears in (32) if it does not vanish.

Remark 5. For the standard case of trigonometric functions $u_{1}(t)=\sqrt{\omega} \cos (\omega t)$ and $u_{2}(t)=\sqrt{\omega} \sin (\omega t), \omega=\frac{2 \pi}{T}$, one can verify using (32) the result as presented in [7] where the case that $T$ tends to zero is considered. However, due to space limitations, we skip the calculations here. Notice also that the related functions $u_{1}(t)=\left(\sin \left(\frac{2 \pi}{T} t\right)\right)^{\frac{1}{2 N+1}}$ and $u_{2}(t)=\left(\cos \left(\frac{2 \pi}{T} t\right)\right)^{2 N+1}$ are a smooth approximation of $u_{1}, u_{2}$ as defined by (7) and (8) for $N \in \mathbb{N}$ sufficiently large.

Remark 6. Theorem 1 is a special case of Theorem 2. This can be seen by evaluating (31) for $u_{1}, u_{2}$ as given by (7) and (8) and using the differentiation property of the state-transition matrix $\Phi$ to get rid of the integral. Notice that $\Phi$ plays the role of $\bar{\Phi}$ as defined in the proof of Theorem 2. By that, one ends up with (16) and following the rest of the proof of Theorem 1 the relation becomes clear.

\subsection{An accelerated gradient algorithm}

In the following we present a new continuous-time optimization algorithm. The algorithm is presented in the following Theorem and is motivated by Lemma 1 as we will explain in Remark 7 .

Theorem 3. Consider

$$
\begin{aligned}
& \dot{z}_{1}=z_{2} \\
& \dot{z}_{2}=-k z_{2}-c_{1} \nabla F\left(z_{1}\right)-c_{2} \nabla F\left(z_{1}+\gamma z_{2}\right)
\end{aligned}
$$

where $k, c_{1}, c_{2}, \gamma>0$ and $z_{1}, z_{2} \in \mathbb{R}^{n}$. Suppose $F: \mathbb{R}^{n} \rightarrow \mathbb{R}$, $F \in \mathscr{C}^{1}$ is locally convex on an open convex set $\mathscr{D} \subseteq \mathbb{R}^{n}$ and has a unique isolated minimum on $\mathscr{D}$ at $z^{*} \in \mathscr{D}$. Then the equilibrium $\left[\begin{array}{ll}z^{* \top} & 0\end{array}\right]^{\top}$ of (42) is locally asymptotically stable. Moreover, if $\mathscr{D}=\mathbb{R}^{n}$ and $F$ is radially unbounded, then the equilibrium is globally asymptotically stable for (42).

Proof. It is straight forward to verify that $\bar{z}_{1}=z^{*}, \bar{z}_{2}=0$ is an equilibrium of (42) since $\nabla F\left(\bar{z}_{1}\right)=\nabla F\left(\bar{z}_{1}+\gamma \bar{z}_{2}\right)=0$. Consider now the Lyapunov function candidate

$$
V=\frac{1}{2} z_{2}^{\top} z_{2}+\left(c_{1}+c_{2}\right) F\left(z_{1}\right)-\left(c_{1}+c_{2}\right) F\left(z^{*}\right)
$$

which is positive definite for $z_{1} \in \mathscr{D}, z_{2} \in \mathbb{R}^{n}$ and attains its minimum at the considered equilibrium. The derivative of $V$ along the trajectories of (42) is given by

$$
\begin{aligned}
\dot{V}= & z_{2}^{\top} \dot{z}_{2}+\left(c_{1}+c_{2}\right) \dot{z}_{1}^{\top} \nabla F\left(z_{1}\right) \\
= & z_{2}^{\top}\left(-k z_{2}-c_{1} \nabla F\left(z_{1}\right)-c_{2} \nabla F\left(z_{1}+\gamma z_{2}\right)\right) \\
& +\left(c_{1}+c_{2}\right) z_{2}^{\top} \nabla F\left(z_{1}\right) \\
= & -k\left\|z_{2}\right\|_{2}^{2}-c_{2} z_{2}^{\top}\left(\nabla F\left(z_{1}+\gamma z_{2}\right)-\nabla F\left(z_{1}\right)\right) .
\end{aligned}
$$

Since $F$ is assumed to be locally convex, we have that

$$
(\alpha-\beta)^{\top}(\nabla F(\alpha)-\nabla F(\beta)) \geq 0 \quad \text { for all } \alpha, \beta \in \mathscr{D} .
$$

Here, $\alpha=z_{1}+\gamma z_{2}$ and $\beta=z_{1}$ such that $\alpha-\beta=\gamma z_{2}$ and hence, since $\gamma>0$, we have $z_{2}^{\top}\left(\nabla F\left(z_{1}+z_{2}\right)-\nabla F\left(z_{1}\right)\right) \geq 0$. Thus, $\dot{V} \leq$ 0 for $z_{1} \in \mathscr{D}, z_{2} \in \mathbb{R}^{n}$. In particular, $\dot{V}=0$ for $z_{2}=0$. However, putting $z_{2} \equiv 0$ into (42), it follows that $z_{1} \equiv z^{*}$ such that by the invariance principle of Krasovskii-LaSalle we conclude that $\left(z^{*}, 0\right)$ is locally asymptotically stable for (42). Equally, in case $\mathscr{D}=\mathbb{R}^{n}, V$ is radially unbounded and $\dot{V} \leq 0$ for all $z_{1}, z_{2} \in \mathbb{R}^{n}$ such that - again by the invariance principle - the equilibrium is globally asymptotically stable.

Remark 7. The algorithm is motivated by the result of Lemma 1 as we will show in the following. Consider again (21) and let $x^{(k)}:=x\left(k \frac{T}{2}\right), k \in \mathbb{N}$. Then we have as a generalization of (21)

$$
x^{(k+2)}=x^{(k)}+\varepsilon^{2} \alpha\left(\frac{\partial F}{\partial x}\left(x^{(k+1)}\right)+\frac{\partial F}{\partial x}\left(x^{(k)}\right)\right)+\mathscr{O}\left(\varepsilon^{3}\right)
$$

where $k \in \mathbb{N}$. With $\xi_{1}^{(k)}:=x^{(k)}, \xi_{2}^{(k)}:=x^{(k+1)}$ we can write this as

$$
\begin{aligned}
\xi_{1}^{(k+1)}-\xi_{1}^{(k)} & =\xi_{2}^{(k)}-\xi_{1}^{(k)} \\
\xi_{2}^{(k+1)}-\xi_{2}^{(k)} & =\xi_{1}^{(k)}-\xi_{2}^{(k)} \\
& +\varepsilon^{2} \alpha\left(\frac{\partial F}{\partial x}\left(\xi_{1}^{(k)}\right)+\frac{\partial F}{\partial x}\left(\xi_{2}^{(k)}\right)\right)+\mathscr{O}\left(\varepsilon^{3}\right) .
\end{aligned}
$$


This is the Euler discretization (with step size 1) of

$$
\begin{aligned}
& \dot{\xi}_{1}=\xi_{2}-\xi_{1} \\
& \dot{\xi}_{2}=\xi_{1}-\xi_{2}+\varepsilon^{2} \alpha\left(\frac{\partial F}{\partial x}\left(\xi_{1}\right)+\frac{\partial F}{\partial x}\left(\xi_{2}\right)\right)+\mathscr{O}\left(\varepsilon^{3}\right) .
\end{aligned}
$$

With $z_{1}:=\xi_{1}, z_{2}:=\xi_{2}-\xi_{1}, c_{1}=c_{2}=\varepsilon^{2} \alpha, \gamma=1$ and neglecting the $\mathscr{O}\left(\varepsilon^{3}\right)$-terms we arrive at (42). Thus, loosely speaking, (42) can be interpreted as the averaged system corresponding to the extremum seeking system discussed in Lemma 1. This relationship suggests that in certain cases extremum seeking algorithms do not mimic a simple gradient flow but the more advanced method presented in Theorem 3.

Remark 8. The continuous-time algorithm (42) can be interpreted as a combination of the continuous-time heavy ball method (see e.g. [3]) and a continuous-time version of Nesterov's method as presented in [6]. In the heavy ball method only the term $\nabla F\left(z_{1}\right)$ is present whereas in Nesterov's method only the term $\nabla F\left(z_{1}+\gamma z_{2}\right)$ occurs. To be more precise, these are given by

$$
\dot{z}_{1}=z_{2} \quad \dot{z}_{2}=u
$$

where $u=-k z_{2}-c_{1} \nabla F\left(z_{1}\right)$ for the heavy ball and $u=-k z_{2}-$ $c_{2} \nabla F\left(z_{1}+\gamma z_{2}\right)$ for Nesterov's method. Notice that if $F$ is quadratic, i.e. the gradient is linear, there is no difference between Nesterov's method and (42) up to the choice of parameters.

Remark 9. The algorithm (42) uses the gradient at two different points which can be interpreted as a simple way of averaging. Moreover, the term $\nabla F\left(z_{1}+\gamma \dot{z}_{1}\right)$ uses knowledge about the derivative of $z_{1}$ which implements some kind of preview. For small $\dot{z}_{1}$, it is also $\nabla F\left(z_{1}+\gamma \dot{z}_{1}\right) \approx \nabla F\left(z_{1}\right)+\gamma \nabla^{2} F\left(z_{1}\right) \dot{z}_{1}$ such that, close to the critical point, this term may also be interpreted as a curvature dependent damping.

\section{Example}

In the following we illustrate the results from Theorem 1 and Theorem 3 by means of an example and simulations.

\subsection{The case of quadratic $F(x)$}

Suppose that $F$ in (6) is a scalar, quadratic function, i.e.

$$
F(x)=x^{2}+b x+c,
$$

where $b, c \in \mathbb{R}$ and $F: \mathbb{R} \rightarrow \mathbb{R}$. The quadratic case is important since many convex functions are locally quadratic in a region around their minimum. For the input sequence defined by (7) and (8) one can compute $x^{*}(t)$ and $\Phi\left(t, t_{0}\right)$ in (10) analytically. In particular, in case of $4 c-b^{2} \neq 0$ one obtains

$$
x^{*}(t)=\frac{1}{2}\left(\tan \left(p\left(t+K_{j}\left(x_{j}\right)\right)\right) \sqrt{4 c-b^{2}}-b\right)
$$

and

$$
\Phi\left(t, t_{j}\right)=\left|\frac{\cos \left(p\left(t_{j}+K_{j}\left(x_{j}\right)\right)\right)}{\cos \left(p\left(t+K_{j}\left(x_{j}\right)\right)\right)}\right|^{2}
$$

for $t \in\left[j T+\varepsilon, j T-\varepsilon+\frac{T}{2}\right]$ with

$$
\begin{aligned}
p & :=\frac{1}{2} \sqrt{4 c-b^{2}} \\
K_{j}\left(x_{j}\right) & :=-j T-\varepsilon+\frac{1}{p} \arctan \left(\frac{b+2 x_{j}}{\sqrt{4 c-b^{2}}}\right) .
\end{aligned}
$$

A derivation of these equations is given in the Section 6. Notice that this also includes the case of $4 c-b^{2}<0$, i.e. $p$ is a complex number, by the definition of the trigonometric functions for complex arguments but we do not discuss this in detail here. The case of $4 c-b^{2}=0$ can be handled similarly and is not treated here as well. Notice further that $x^{*}(t)$ has finite escape time at $t_{\text {esc }, j}=\frac{2 m+1}{2 p} \pi-K_{j}\left(x_{j}\right)$ with $m \in \mathbb{Z}$. However, in most cases this is no problem in the implementation of the approximation from Theorem 1. We implemented the iteration

$$
\begin{aligned}
x((j+1) T) & =x(j T) \\
& +\varepsilon \alpha \Phi(j T, j T+\varepsilon)\left(1-\Phi\left(j T+\varepsilon, j T+\frac{T}{2}-\varepsilon\right)\right)
\end{aligned}
$$

which is another representation of (20) and where we neglected the higher order terms. Thus, if $t_{e s c, j} \neq j T$ and $t_{e s c, j} \neq j T+\varepsilon$, the evaluation makes no problem. As shown in the Section 6, the iteration (54) has fix points at

$$
\bar{x}_{1 / 2}=\frac{1}{2}\left(\sqrt{4 c-b^{2}} \frac{\cos \left(\frac{p T}{2}-2 \varepsilon\right) \mp 1}{\sin \left(\frac{p T}{2}-2 \varepsilon\right)}-b\right) .
$$

Notice that the minimum of the function $F=x^{2}+b x+c$ is at $x_{\text {min }}=-\frac{b}{2}$. Thus, since $\cos \left(\frac{p T}{2}-2 \varepsilon\right) \mp 1 \neq 0$ for $\sin \left(\frac{p T}{2}-2 \varepsilon\right) \neq$ 0 , it is $\bar{x}_{1 / 2} \neq x_{\min }$ such that the iteration never converges to the minimum of $F$. However, we can get arbitrarily close. Secondly, it is worth to mention that (54) possesses two fix points. Simulations suggest that one is asymptotically stable and the other one is unstable where the stability depends on the chosen period length $T$.

In our simulations we compared the approximative iteration (54) with a fixed step simulation of the original system, i.e. (6) with inputs (7) and (8), and a simple gradient descent. The simulation results are depicted in Figure 4. At the respective time points $j T$ our approximation is much closer to the original system as the gradient descent which seems to correspond to an average of the original system. This is to be expected since here the period length is large compared to the length of the needle which is also the reason for the large jumps to be seen on the right hand side of Figure 4. In the considered example our approximation seems to be a good measure for the lower "boundary" of the trajectory of the original system. Notice that also an approximation of the upper boundary can be computed similarly. 

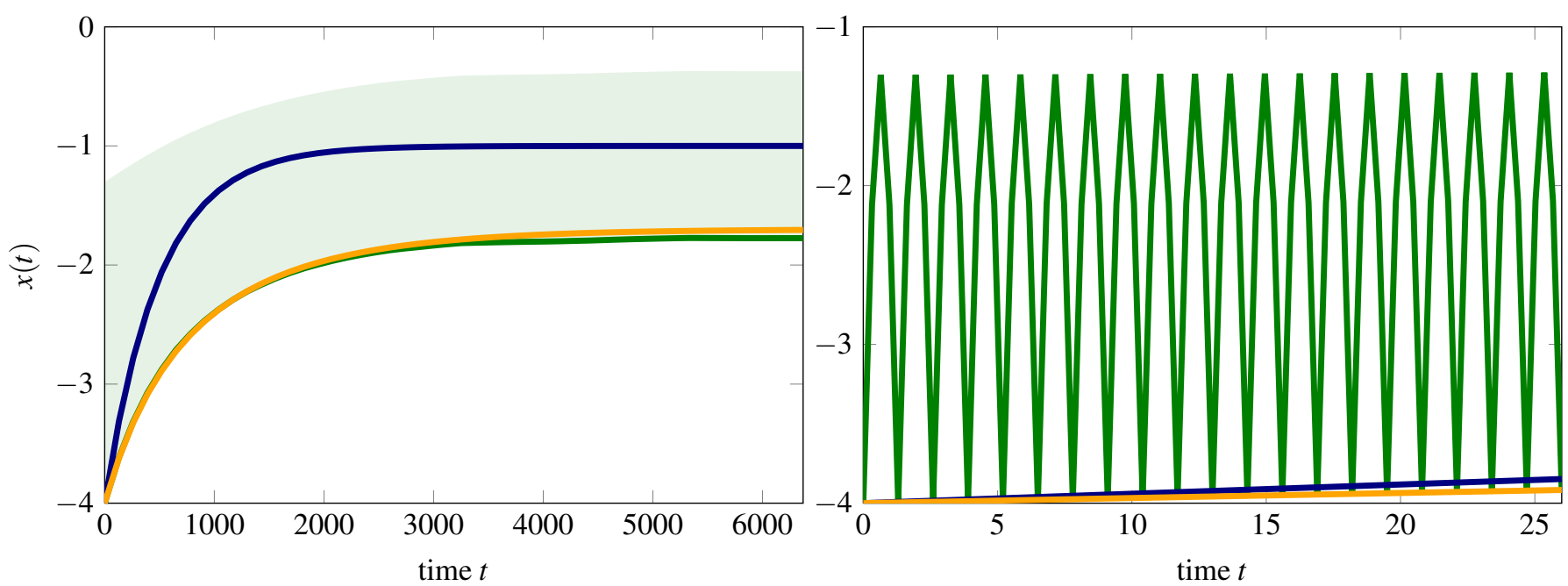

Figure 4. A comparison between the original system (green), i.e. system (6) with inputs (7) and (8), the approximation (10) (orange) and a gradient descent algorithm (blue) in the case of quadratic $F(x)\left(b=2, c=3, \varepsilon=0.00001, T=1.3, x_{\min }=-1, \alpha=-10\right)$. On the left hand side the thick green line depicts the original system evaluated at multiples of the period length; the shaded area shows the original system jumping up and down very fast. A zoomed plot that illustrates this jumping can be seen on the right hand side.

\subsection{Simulative analysis of the algorithm (42)}

We compared the proposed algorithm (42) to the heavy ball method and a continuous-time version of Nesterov's method (see also Remark 8). Simulation results for the case of $F(x)=|x|^{3}$ are depicted in Figure 5. In the considered case the proposed algorithm shows a fast convergence without overshoot in comparison to both other algorithms. Further simulations with different objective functions or varied parameters show a similar behavior. However, the simulation results should be interpreted with care when it comes to performance or convergence speed since all three algorithms include parameters and it is not clear how to choose them in a way such that direct comparisons are possible.

\section{Conclusions and outlook}

In this work we introduced needle-shaped dither signals for gradient approximation and extremum seeking. We derived formulas that give insight into the averaging process of extremum seeking schemes with needle-shaped dither signals. We further showed how this can be generalized to arbitrary periodic dither signals by superposition. Thus, needle-shaped dithers can be seen as basis functions for a wide range of more general signals. Motivated by these results we also proposed a new gradient-based optimization algorithm. The algorithm is related to well-known accelerated gradient methods and is of interest on its own. By taking two gradients into account the behavior of the proposed algorithm is similar to the averaged behavior of the extremum seeking scheme with needle-shaped dither signals. This might be one hint why extremum seeking schemes often perform relatively well in practice despite their simplicity. Since our approach relies on well-established ideas from the

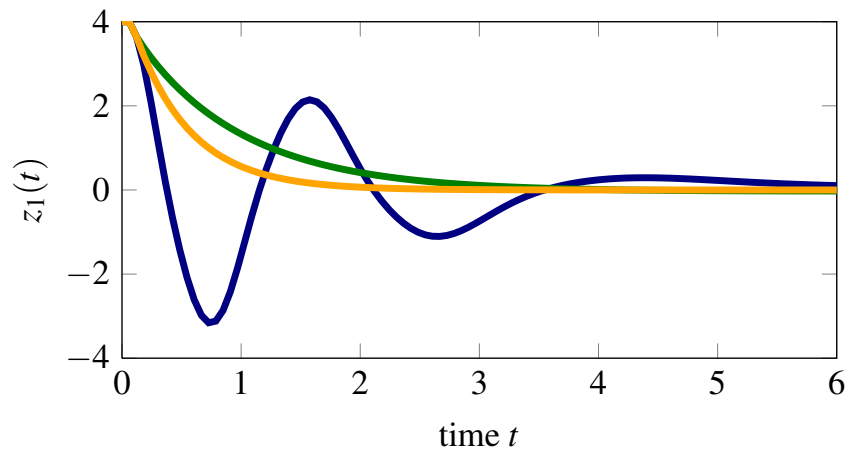

Figure 5. Comparison of the heavy ball method (blue), Nesterov's method (green) and the proposed algorithm (42) (orange) for the minimization of $F(x)=|x|^{3}$. The parameters of the new algorithm are chosen as $c_{1}=c_{2}=\gamma=1, K=2$. For Nesterov's method they are chosen such that for the case of quadratic $F(x)$ it is equal to the proposed algorithm. The numerical integration is done using a standard fixed step Euler method.

Maximum Principle we hope that we can extend our setup using existing generalizations of the Maximum Principle ([14]). As already mentioned before, we also expect that our results can be extended to the multidimensional case. Moreover, we aim to use our new knowledge about the gradient approximation process to design dither signals that are in some terms optimal.

\section{References}

[1] Milton Abramowitz, Irene A. Stegun, et al. Handbook of mathematical functions, volume 1. Dover New York, 1972. 
[2] Kartik B. Ariyur and Miroslav Krstic. Real-time optimization by extremum-seeking control. John Wiley \& Sons, 2003.

[3] Hedy Attouch, Xavier Goudou, and Patrick Redont. The heavy ball with friction method, I. The continuous dynamical system. Communications in Contemporary Mathematics, 2(01):1-34, 2000.

[4] Roger W. Brockett. Nonlinear systems and differential geometry. Proceedings of the IEEE, 64(1):61-72, Jan 1976.

[5] Pascal Cougnon, Denis Dochain, Martin Guay, and Michel Perrier. On-line optimization of fedbatch bioreactors by adaptive extremum seeking control. Journal of Process Control, 21(10):1526-1532, 2011.

[6] Hans-Bernd Dürr, Erkin Saka, and Christian Ebenbauer. A smooth vector field for quadratic programming. In $D e$ cision and Control (CDC), 2012 IEEE 51st Annual Conference on, pages 2515-2520, Dec 2012.

[7] Hans-Bernd Dürr, Milos S. Stankovic, Christian Ebenbauer, and Karl Henrik Johansson. Lie bracket approximation of extremum seeking systems. Automatica, 49(6):1538 $-1552,2013$.

[8] Thomas Kailath. Linear Systems. Prentice-Hall, 1980.

[9] Hassan K. Khalil. Nonlinear Systems. Prentice-Hall, 2002.

[10] Jaroslav Kurzweil and Jiří Jarník. Limit processes in ordinary differential equations. Zeitschrift für angewandte Mathematik und Physik ZAMP, 38(2):241-256, 1987.

[11] Daniel Liberzon. Calculus of variations and optimal control theory: A concise introduction. Princeton University Press, 2011.

[12] S. Michalowsky and C. Ebenbauer. Gradient approximation and extremum seeking via needle variations. In American Control Conference (ACC), 2016 (to appear), 2016.

[13] James C. Spall. Multivariate stochastic approximation using a simultaneous perturbation gradient approximation. Automatic Control, IEEE Transactions on, 37(3):332-341, 1992.

[14] Héctor J. Sussmann. Needle variations and almost lower semicontinuous differential inclusions. Set-valued analysis, 10(2-3):233-285, 2002.

[15] Ying Tan, Dragan Nesic, and Iven Mareels. On the choice of dither in extremum seeking systems: A case study. Automatica, 44(5):1446 - 1450, 2008.

[16] William F. Trench. Introduction to real analysis. Prentice Hall/Pearson Education Upper Saddle River, NJ, 2003.

[17] Hsin-Hsiung Wang, Simon Yeung, and Miroslav Krstic. Experimental application of extremum seeking on an axialflow compressor. Control Systems Technology, IEEE Transactions on, 8(2):300-309, 2000.

[18] Chunlei Zhang and Raúl Ordóñez. Extremum-seeking control and applications: A numerical optimization-based approach. Springer, 2011.

\section{Appendix}

\subsection{Derivation of (50), (51)}

For $F$ as defined by (49) and $t \in\left[j T+\varepsilon, j T-\varepsilon+\frac{T}{2}\right], j \in \mathbb{N}$, $x^{*}(t)$ is the solution of

$$
\dot{x}^{*}(t)=x^{* 2}(t)+b x^{*}(t)+c, \quad x(j T+\varepsilon)=: x_{j} .
$$

We will now solve this differential equation via separation of variables. Resorting and integrating gives

$$
\int_{x_{j}}^{x^{*}} \frac{1}{\xi^{2}+b \xi+c} d \xi=\int_{j T+\varepsilon}^{t} 1 d \tau
$$

These integrals can be solved using standard formulas for elementary functions, see e.g. [1]. If $4 c-b^{2} \neq 0$ we have that

$$
\left[\frac{2}{\sqrt{4 c-b^{2}}} \arctan \left(\frac{b+2 \xi}{\sqrt{4 c-b^{2}}}\right)\right]_{x_{j}}^{x^{*}}=t-j T-\varepsilon .
$$

Notice that this also includes the case of $4 c-b^{2}<0$ by the definition of the $\arctan \operatorname{as} \arctan (z)=\frac{i}{2} \ln \left(\frac{i+z}{i-z}\right)$ (see [1]) where $i=\sqrt{-1}$ is the imaginary unit. Thus, solving this equation for $x^{*}$ we obtain the solution as given by (50). To obtain the transition matrix $\Phi\left(t, t_{j}\right)$ we use that the variational equation (9) here is a scalar linear time varying differential equation such that (see e.g. [8]) $\Phi\left(t, t_{j}\right)=\exp \left(\int_{t_{j}}^{t} \frac{\partial F}{\partial x}\left(x^{*}(\tau)\right) d \tau\right)$. Putting the solution (50) into this equation we obtain

$$
\begin{aligned}
\Phi\left(t, t_{j}\right) & =\exp \left(\int_{t_{j}}^{t} \sqrt{4 c-b^{2}} \tan \left(p\left(\tau+K_{j}\left(x_{j}\right)\right)\right) d \tau\right) \\
& =\exp \left(\sqrt{4 c-b^{2}}\left[-\frac{\ln \left(\left|\cos \left(p\left(\tau+K_{j}\left(x_{j}\right)\right)\right)\right|\right)}{p}\right]_{t_{j}}^{t}\right) \\
& =\left|\frac{\cos \left(p\left(t+K_{j}\left(x_{j}\right)\right)\right)}{\cos \left(p\left(t_{j}+K_{j}\left(x_{j}\right)\right)\right)}\right|^{-2} .
\end{aligned}
$$

\subsection{Fix points of the iteration (54)}

We briefly analyze the iteration (54). Let $t_{j 1}:=j T+\varepsilon$ and $t_{j 2}:=j T+\frac{T}{2}-\varepsilon$. Since $\varepsilon>0, \alpha>0,(54)$ has a fix point at $\bar{x}$ implicitly given by the equation

$$
1-\Phi\left(t_{j 1}, t_{j 2}\right)=1-\left|\frac{\cos \left(p\left(t_{j 2}+K_{j}(\bar{x})\right)\right)}{\cos \left(p\left(t_{j 1}+K_{j}(\bar{x})\right)\right)}\right|^{2}=0 .
$$

By (51) we have with (52) and (53)

$$
\Phi\left(t_{j 1}, t_{j 2}\right)=\left|\frac{\cos \left(\frac{p T}{2}-2 p \varepsilon+\arctan \left(\frac{b+2 x_{j}}{\sqrt{4 c-b^{2}}}\right)\right)}{\cos \left(\arctan \left(\frac{b+2 x_{j}}{\sqrt{4 c-b^{2}}}\right)\right)}\right|^{2}
$$


and using the trigonometric identity

$$
\frac{\cos (\alpha+\beta)}{\cos (\beta)}=\cos (\alpha)+\sin (\alpha) \frac{\sin (\beta)}{\cos (\beta)}
$$

we obtain

$$
\Phi\left(t_{j 1}, t_{j 2}\right)=\left|\cos (\alpha)-\sin (\alpha) \frac{b+2 x_{j}}{\sqrt{4 c-b^{2}}}\right|^{2}
$$

with $\alpha:=\frac{p T}{2}-2 p \varepsilon$. We compute further

$$
\begin{aligned}
& 1-\Phi\left(t_{j 1}, t_{j 2}\right) \\
= & 1-\cos ^{2}(\alpha)+2 \sin (\alpha) \cos (\alpha) \frac{\alpha}{\sqrt{4 c-b^{2}}}+\sin ^{2}(\alpha) \frac{\left(b+2 x_{j}\right)^{2}}{4 c-b^{2}} \\
= & \sin ^{2}(\alpha)\left(1-\frac{\left(b+2 x_{j}\right)^{2}}{4 c-b^{2}}\right)+2 \sin (\alpha) \cos (\alpha) \frac{b+2 x_{j}}{\sqrt{4 c-b^{2}}}
\end{aligned}
$$

In the following we assume that $\sin (\alpha) \neq 0$. Then, using the previous results, the equation for the fix point is given by

$$
\sin (\alpha)\left(1-\frac{(b+2 \bar{x})^{2}}{4 c-b^{2}}\right)+2 \cos (\alpha) \frac{b+2 \bar{x}}{\sqrt{4 c-b^{2}}}=0
$$

Let $w:=\frac{(b+2 \bar{x})}{\sqrt{4 c-b^{2}}}$. Then this is a quadratic equation in $w$ and its solutions are given by $w_{1 / 2}=\frac{\cos (\alpha) \mp 1}{\sin (\alpha)}$. Thus, with $\alpha=\frac{p T}{2}-$ $2 p \varepsilon$, the fix points are given as in (55). 